\title{
La gazette nationale de Saragosse
}

Entre collaboration et Afrancesamiento

\section{Frédéric Dauphin}

\section{(2) OpenEdition}

Journals

Édition électronique

URL : https://journals.openedition.org/ahrf/1704

DOI : 10.4000/ahrf.1704

ISSN : 1952-403X

Éditeur :

Armand Colin, Société des études robespierristes

Édition imprimée

Date de publication : 1 juin 2004

Pagination : 147-168

ISSN : 0003-4436

\section{Référence électronique}

Frédéric Dauphin, « La gazette nationale de Saragosse », Annales historiques de la Révolution française [En ligne], 336 | avril-juin 2004, mis en ligne le 15 juillet 2007, consulté le 22 avril 2022. URL : http:// journals.openedition.org/ahrf/1704; DOI : https://doi.org/10.4000/ahrf.1704

Ce document a été généré automatiquement le 22 avril 2022.

Tous droits réservés 


\title{
La gazette nationale de Saragosse
}

\author{
Entre collaboration et Afrancesamiento
}

\author{
Frédéric Dauphin
}

1 À ceux qui s'interrogeaient sur l'intérêt de la presse du Consulat et de l'Empire en opposant le talent de sa devancière révolutionnaire à sa docilité toute impériale, André Cabanis avait répondu que : « c'est par son abaissement même que la presse du premier Empire valait d'être étudiée $»^{1}$.

2 C'est donc à l'analyse d'un double abaissement que nous nous attacherons, puisqu'à sa fonction propagandiste initiale, la gazette nationale de Saragosse joignait son opposition au soulèvement national des Espagnols contre l'oppresseur français. Traitre à la cause résistante, elle prenait fait et cause pour une modernisation de l'Espagne contre les Espagnols, par adhésion à un régime et à des idées venues d'outre Pyrénées. Avec ses consœurs de la dénommée presse "afrancesada», elle allait inonder les principales villes de la moitié Nord du pays pour faire contre-poids durant toute la Guerre d'Indépendance à la presse résistante espagnole.

La qualité comme la quantité des gazettes de collaboration fluctua ainsi au gré des avancées ou des défaites françaises dans une sorte de "guerre des presses", à la fois modèle réduit et synthèse écrite au jour le jour de ladite Guerre d'Indépendance. Car voilà l'atout majeur de telles sources, à la fois actrices de l'histoire et reflet de l'engagement politique quotidien de leurs auteurs afrancesados, loin des jugements a posteriori que la seule analyse critique de leurs divers Mémoires nous imposait jusque là.

Listées par Gomez Imaz au nombre de 17, ces gazettes seraient en réalité $32^{2}$, dispersées entre l'Hémérothèque de Madrid, la Bibliothèque Nationale d'Espagne et diverses archives régionales. Sujettes à quelques analyses individuelles ${ }^{3}$, elles n'ont toujours pas fait l'objet d'une étude de synthèse que leur éparpillement, la faiblesse des archives annexes éventuelles et leur nombre aléatoire suivant les années, rendent difficile. $\mathrm{Ne}$ pouvant rendre compte d'un travail qui n'en est qu'à ses balbutiements, je me limiterai à l'analyse de l'une d'entre elles : la gazette nationale de Saragosse. Il s'agira, fort succinctement, d'aller de la gazette à l'idéologie afrancesada en étudiant cette dernière comme un outil de propagande avec ses méthodes et plus encore ses dysfonctionnements, puis comme un symbole de ré-appropriation de l'idéal ilustrado 
par ses auteurs et enfin comme un moyen d'action au jour le jour de l'afrancesamiento avec son corollaire de questions faisant débat et de champs d'investigations non encore explorés.

5 Fille non reconnue de la toute jeune et pourtant foisonnante histoire du journalisme aragonais des années de l'Ilustracion, la gazette nationale de Saragosse allait tenter de fusionner de 1809 à 1812 les acquis de son passé ilustrado avec les exigences d'un journal officiel napoléonien. Mais relevant l'impossible combat de concilier l'inconciliable, trop propagandiste pour les Espagnols, trop proche des Lumières ibériques et du souvenir de l'Ilustracion de Charles III pour les généraux français, la gazette se cherchera en vain un lectorat que son adversaire de la résistance sut trouver autour du Roi et de la Foi.

Caractéristiques générales

6 Si nous nous référons aux deux seuls historiens traitant de la gazette nationale de Saragosse, à l'exception de Gomez Imaz ${ }^{4}$, celle-ci a peu d'intérêt eu égard à ses concurrentes résistantes. Faite selon eux : "au goût de son inspirateur», sans que l'on sache s'il s'agit de Napoléon, de Suchet ou du rédacteur Ased, cette gazette ne serait qu'un détail de la jeune et pourtant riche histoire du journalisme aragonais. Or, loin d'une mise entre parenthèse $d u$ journalisme espagnol durant l'occupation napoléonienne, nous verrions plutôt dans la parution de la gazette nationale de Saragosse les signes d'une continuité journalistique régionale avec ses deux illustres prédécesseurs : la Gazeta et le Diaro de Saragosse.

7 Bien que les archives nous offrent un nombre conséquent de gazettes, nous ne disposons pas des premiers numéros. Précédée par un journal rédigé par les militaires français, le Prontuario nacional, la gazette nationale de Saragosse serait selon Antonio Duenas Labarias apparue le 20 avril 1809 et imprimée chez : «los herederos de francisco Moreno ». Pour notre part, en l'absence d'une gazette datant d'avril 1809 ou d'archives relatant son existence, nous nous fonderons sur la date du 14 août 1809 que nous retrouvons dans les comptes de l'administrateur Sevil concernant la paye de son rédacteur et les coûts de fabrication du périodique. Une telle date en cours de mois d'août comme base comptable n'est certainement pas sans signification et correspond si ce n'est au début officiel du journal, du moins à un changement de rédacteur, voire à des modifications de statuts ou d'objectifs.

De caractère officiel, ce bi-hebdomadaire (jeudi et dimanche) se vendait soit « $a$ la menuda » soit par abonnements. Mais l'essentiel des tirages était probablement destiné aux souscriptions obligatoires de chaque pueblos d'Aragon, transformant la gazette en véritable journal officiel. Toujours d'après les comptes, un seul rédacteur rétribué était recensé sous le nom de Miguel Ased. Au-dessus de lui, un administrateur, Agustin Sevil, chargé de maintenir "sous perfusion" une gazette très rapidement déficitaire.

Composée en moyenne de 4 pages (25X17), la gazette pouvait atteindre les 6 voire 8 pages si un décret important de Suchet y était inséré. Chaque gazette était numérotée à partir du début de l'année civile. En dessous de ce numéro et encadré par deux traits, le titre en majuscule taille 12 de "GAZETA NACIONAL DE ZARAGOZA" avec le jour de parution. Puis la gazette se scindait en deux avec (sur 5 lignes environ) les "afecciones astrologicas » d'un côté et "meteologicas» de l'autre. Enfin, le texte de taille 10 s'étalait sur 2 colonnes sans séparation. Chaque gazette se concluait par une phrase en bas de la dernière page en gros caractère "DE ORDEN DEL GOBIERNO ; EN LA IMPRENTA DEL HOSPITAL $»^{5}$. 
10 En terme de contenu, la gazette comme ses consœurs européennes passait allègrement des affaires internationales aux affaires nationales et enfin locales, sans toutefois que certains décrets ou textes d'importances ne viennent épisodiquement déranger ce parfait enchaînement rédactionnel. Les "noticias extrangeras", en première page, reprenaient en écho les propos du Moniteur mais aussi de la Gazette de Madrid ou de gazettes afrancesadas provinciales avec un mois et demi de retard en moyenne. La Russie, l'Allemagne, et surtout l'Empire français étaient les plus présents au sein de comptes rendus oscillant entre 6 et 35 à 40 lignes suivant le pays et l'intérêt du sujet. Puis les affaires d'Espagne ou d'Aragon constituaient les pages névralgiques de la gazette en tant qu'outil de propagande. Les affaires locales et culturelles ponctuaient le journal, signe que malgré la guerre la vie continuait. Les variétés (variedades), les " anuncios » et le théatre servaient, à cet effet, de base coutumière à la dernière page, voire aux deux dernières en fonction des périodes d'accalmie où la propagande basique pouvait laisser place à un afrancesamiento plus culturel à connotation politique.

La gazette nationale de Saragosse, un outil de propagande.

11 Fort de ses expériences passées en France comme dans le reste de l'Europe, Napoléon tenta très tôt d'associer à l'occupation du territoire espagnol une logique propagandiste diffusée à travers la création d'une presse nationale capable de participer au débat politique naissant lors de la Guerre d'Indépendance. En tant que presse propagandiste dite d'intégration ${ }^{6}$, la presse afrancesada cherchait à développer la volonté d'une unanimité idéologique et de participation de tous au nouveau régime. Pour y arriver, quatre graduations ou stades étaient nécessaires, allant de la simple recherche de l'unanimité autour d'un nom ou d'un Roi jusqu'au comportement conforme au désir recherché. Il fallait prioritairement un changement du milieu ambiant jusqu'à ce que disparaissent tous les groupes anciens synonymes du système précédent. Le passé révolu, le système devait conduire ses lecteurs au refus de l'idéologie des insurgés et à leur intégration complète au sein du nouveau régime.

12 Mais la réalité fut bien différente. Le passage du rejet à l'intégration des lecteurs dans le système du gouvernement «intruso " devait s'avérer plus difficile que prévu. Non seulement à cause des résistances externes au système napoléonien, mais surtout du fait des incohérences internes propres au système propagandiste. Car si les graduations proposées par J. J. Aranda se retrouvent effectivement dans notre gazette, elles n'ont pas une logique globale aussi affirmée qu'on pourrait le croire. Pourquoi ? Tout simplement parce que la gazette n'était pas le produit d'une propagande monolithique, mais de trois propagandes simultanées.

13 Celle de Suchet bien évidemment, principalement répressive (et, ou administrative), essentiellement d'envergure locale et axée sur les résistants aragonais. Celle de Joseph et des afrancesados ensuite (auxquels nous adjoignons notre rédacteur), d'échelle nationale et ayant pour but l'intégration des Espagnols au sein du nouveau régime dans une sorte de résurgence tardive de l'Ilustracion. Celle de Napoléon enfin, d'envergure internationale, relayée dans toute la presse de l'Empire et de caractère singulièrement charismatique.

$\mathrm{Du}$ caractère triple de cette propagande allait résulter des enchevêtrements et plus encore des incohérences dans le discours global, expliquant en partie l'échec de ce système propagandiste.

D'abord gérer le combat quotidien. 

d'abord dans la lutte propagandiste contre le système de guérillas que cette gazette trouvera les raisons de son maintien sous perfusion financière ${ }^{7}$. Bien que fluctuants au gré de la pacification de la région, les articles relatifs à la résistance spécifiquement aragonaise constitueront, pour l'année 1809 et le premier semestre 1810, le cœur même de la gazette, éclipsant par leur quantité les articles relatifs aux affaires internationales ou aux variedades. Toutefois, de résistance il n'est quasiment jamais fait mention. Comme pour la gazette de Navarre (étudiée par J. J. Sanchez Aranda), celle de Saragosse niait ce statut à ceux qui combattaient le gouvernement militaire du maréchal Suchet. Ainsi les résistants locaux se voyaient-ils accolés les attributs de bandits ou de voleurs dans le cadre d'un simple problème d'ordre ou plutôt de désordre public. La raison en était simple. Ne pas qualifier les adversaires de résistants revenait en fait à nier leur unité tout en minimisant leur champ d'action'.

Toutefois, les actions de la résistance locale ne pouvaient rester impunies. De multiples décrets de Suchet usèrent à cet effet des méthodes classiques du dénigrement méthodique et répétitif. Ainsi, aux condamnations collectives des villages ayant collaboré avec les "brigands", répondait quotidiennement l'éloge de ceux ayant fait preuve de fidélité au gouvernement. Or, si l'association de villages avec les insurgés entraînait systématiquement des condamnations financières fortes, Suchet offrait, à l'inverse, des récompenses financières aux populations qui collaboraient avec lui. Toujours dans le cadre d'une stratégie d'alternance du "bâton et de la carotte", le gouverneur d'Aragon, pour répondre au système de contrebande né du blocus continental, organisait un système de délation rétribuée dont la gazette servait à la fois d'organe d'émission comme de réception. En tant que périodique officiel, la gazette devait être achetée par chaque «pueblo» d'Aragon. C'est donc tout naturellement qu'elle revêtait les obligations d'un journal officiel à destination des municipalités avec celles plus basiques d'un outil propagandiste à l'intention d'une population paysanne trop sensible au discours résistant.

17 D'un point de vue plus strictement militaire, la désertion ou le passage à l'ennemi comme le douloureux problème de l'approvisionnement quotidien furent, eux aussi, sujets d'innombrables textes officiels, comme autant de garanties offertes par Suchet aux marchands et commerçants saragossains. À n'en point douter, le devoir d'exemplarité militaire des troupes impériales n'était pas gratuit puisque corrélatif de la pacification, à terme, de la région.

sure le désordre aragonais, ce premier niveau de propagande émanait directement de Suchet. D'aspect exclusivement répressif, il répondait avant tout à la prolifération des actions de la résistance locale en ouvrant des "contre-feux" par le recours aux pratiques du dénigrement, des condamnations ou de l'exemplarité civile ou militaire. Contrebalançant les actes de la résistance, il n'avait pas d'objectif à moyen ou long terme, mais juste une obligation de répondre à l'adversaire pour ne pas laisser une impression de délitement du système napoléonien aux Aragonais. Toutefois cette propagande "au jour le jour" de Suchet se doublait dans certains cas d'une campagne démobilisatrice plus complexe prenant plusieurs gazettes pour supports et dépassant le simple cadre répressif pour tendre vers la logique de l'adhésion forcée.

19 Là encore, le style très militaire ne cherchait pas à démontrer le pourquoi ou les intérêts de chacun à une telle soumission, mais plutôt son caractère obligatoire, tant la victoire française paraissait imprégnée du sceau de la puissance. Ainsi, les grandes

Annales historiques de la Révolution française, 336 | avril-juin 2004 
victoires continentales de l'empereur se transformaient, à l'instar du journal d'Italie, en autant de moment d'anthologie littéraire digne d'Hérodote ou de Thucydide. De la même manière, la multiplicité des passages de troupes françaises à la frontière (croisant de non moins nombreux convois de prisonniers) justifiait le caractère irréversible des victoires françaises par l'allégorie de la mère patrie nourricière inépuisable en ressources matérielles et humaines'. D'autres fois, afin d'accentuer ce côté irrémédiable, le journal reprenait des lettres de soi-disant prisonniers espagnols qui, écrivant à leurs femmes pour les rassurer sur leur état de santé, faisaient d'une soumission totale au nouveau régime la condition sine qua non d'un possible retour au foyer.

Parfait exemple d'une gazette concentrant tous les arguments ou presque de la propagande napoléonienne en une suite logique d'événements et d'auteurs, celle-ci conduisait tour à tour le lecteur à se soumettre d'abord par la force des choses et des armes françaises, ensuite par l'incompétence, la nullité et les mensonges d'une résistance manipulée par l'Angleterre et enfin, pour la quête d'une paix préservant «les intérêts généraux de la patrie et les intérêts particuliers de chaque habitant ". Cette évolution $\mathrm{du}$ discours vers une vision de plus en plus "positive", par l'acceptation de tous de l'ordre établi, dépassait donc le cadre local et l'actualité immédiate pour tendre vers des objectifs à moyen terme de soumission volontaire.

Bien que plus astucieuse et réfléchie, cette propagande provenant du gouvernement militaire aragonais ne pouvait cependant suffire à convaincre les Espagnols sur la longue durée. La soumission face au grand Napoléon ne pouvant à elle seule constituer un projet d'avenir, il convenait d'évoluer petit à petit vers une autre politique propagandiste dite d'intégration ou de communion au projet «joséphinide ».

Du principe de rejet à celui de l'adhésion.

Cette propagande, de caractère national et relayée par toutes les gazettes d'occupation, par la reprise des articles de la gazette de Madrid, prêchait l'adhésion au nouveau régime non plus par obligation, mais par intérêt. Parmi les moyens employés, le premier qui nous vient à l'esprit est celui de la récupération politique des anciens résistants ${ }^{10}$. Le stratagème se voulait novateur et insidieux puisque s'appuyant sur la faiblesse ou la suspicion de faiblesse de ceux qui combattaient aux côtés des insurgés. À mi-chemin entre la terreur de la propagande militaire locale et l'idéal ilustrado dont feront preuve les afrancesados à partir de 1811, cette politique devait certainement correspondre à une époque de transition, du moins dans l'esprit de ses auteurs. Outre les militaires, les ecclésiastiques étaient mis à contribution par la propagande strictement joséphinide, non seulement pour limiter l'intervention des "soldats" de l'Église dans le combat résistant, mais surtout dans le but de soutenir le nouveau régime. À titre d'exemple, l'allusion répétée aux vieilles monarchies de droit divin légitimant les changements dynastiques proposait aux Espagnols non plus une soumission face à la force de l'occupant, mais une adhésion au nouveau régime par la soumission aux volontés de la sainte religion. Il en est ainsi de l'ambiguité d'une propagande aux contradictions permanentes où le recours aux valeurs d'Ancien Régime pouvait s'avérer parfois bien plus efficace qu'un discours plus progressiste. Joseph et les afrancesados donnaient ici aux Espagnols indécis ou passifs un discours répondant à leur spécificité culturelle, ce que l'unicité de la propagande napoléonienne émanant des Tuileries ne fera jamais. était racontée par la gazette de façon régulière et suivant un schéma préétabli relevant, 
là encore, du feuilleton bi-hebdomadaire. À chaque fois, l'auteur débutait en citant le nom de la ville qui avait eu l'honneur de recevoir le "bon roi Joseph", puis reprenait les actions et autres allocutions du souverain. Il était question de régénérer l'Espagne, de la sortir de son archaïsme et le premier pas qui devait l'y mener était l'acceptation par tous de ce nouveau et grand roi. Malheureusement, l'entreprise s'avérerait ardue tant l'image de Ferdinand, savamment entretenue par l'élite résistante, offrait un adversaire difficile à combattre. En outre, l'émergence de Ferdinand dans le discours propagandiste était symptomatique d'un discours pluriel, où la propagande strictement napoléonienne pouvait parfois aller à l'encontre même des intérêts de Joseph et de son gouvernement.

Napoléon ou les limites de la propagande impériale.

24 Napoléon, comme nous le savons, contrôlait ou faisait contrôler tout ce qui touchait à son système de propagande, allant parfois jusqu'à indiquer le style, la présentation et le contenu des textes que l'on devait retrouver un peu plus tard dans toutes les gazettes de l'hexagone, d'Espagne, voire de l'Empire ${ }^{11}$. Conscient de l'impact de la presse sur l'opinion publique, mais plus conscient encore de l'instabilité de son régime et de l'incapacité de Joseph à régner sur une Espagne en ébullition permanente, l'empereur, en réintroduisant "médiatiquement" Ferdinand dans le jeu politique espagnol, se réservait, vraisemblablement, une porte de sortie à plus ou moins brève échéance avec le retour de ce roi potentiel. J'en veux pour preuve le traitement de l'affaire dite « de l'enlèvement de Valençay » ou la campagne de discrédit fut exclusivement orientée sur l'Angleterre et non sur Ferdinand ${ }^{12}$. Étant reprise dans tous les journaux de l'Empire, il est évident que cette information résultait de l'intervention directe de Napoléon au risque d'anéantir la stratégie de l'omission pure et simple appliquée jusque là par les rédacteurs josephinides envers Ferdinand.

D'apparence charismatique, la propagande napoléonienne n'était ni une idée ni une doctrine, mais un homme : Napoléon. En tant que dernier niveau de propagande, ce discours provenait directement des Tuileries et incarnait "au mot près" la pensée de son chef. Mais cette pensée n'avait que peu d'attirance pour Ferdinand et ses compatriotes, car concrètement bien plus anti-britannique que réellement espagnole. Ainsi, l'Angleterre, en tant qu'acteur central du conflit espagnol et obsession première de l'empereur, allait se retrouver à de multiples reprises, soit à travers des textes condamnant les activités britanniques, soit dans la publication d'extraits du Mirror ou du Daily telegraph ou de soi-disantes lettres interceptées d'agents ou militaires anglais. Or, si le rapport singulier et historique de l'Espagne à l'espace atlantique ou bien encore le rôle prépondérant de l'Angleterre dans la résistance ibérique suffisent à expliquer ces attaques, elles n'en justifient pas la primauté. Cherchons donc plutôt dans cet acharnement systématique le reflet d'une vision monochrome et uniforme qu'avait Napoléon des Européens. Apparaissent ainsi dans cette façon d'aborder le problème espagnol aussi bien les limites de son système de propagande que celles de l'homme Napoléon. Cherchant à souder les Européens dans une opposition simpliste et caricaturale à l'Angleterre, il négligeait les spécificités culturelles et historiques de chaque nation et de chaque membre qui la compose.

26 Cependant, les raisons d'un échec journalistique comme le fut celui de la presse afrancesada ne tenait pas, à l'instar des batailles, uniquement aux erreurs de jugement de l'empereur, mais plutôt à une série de dysfonctionnements internes rendant la copie bi-hebdomadaire presque illisible. 

discours dans un seul et même organe de presse. À chaque fonction ou attribut confiés à la gazette correspondait un niveau de lecture et par conséquent de lecteurs. Les objectifs parfois convergents des acteurs du journal (Napoléon, Joseph, les afrancesados et Suchet) pouvaient à d'autres moments s'avérer en opposition totale. Plus d'une fois, les nécessités d'une propagande censée impressionner les résistants par le caractère implacable du pouvoir militaire de Napoléon furent en contradiction avec un discours beaucoup plus afrancesado de régénération et de pacification incarné par Joseph. L'image même d'un Napoléon libérateur et en même temps " Dieu vivant de la guerre " dut laisser dubitatif plus d'un lecteur érudit.

Illisible, ensuite, par la multiplicité de ses fonctions comme de ses objectifs. Tour à tour journal de propagande et de combat contre les résistants, mais aussi journal de proximité et de "néo Ilustracion» culturelle, la gazette ne put offrir de ligne rédactionnelle claire à même de s'attirer un lectorat régulier. A fortiori par rapport à sa devancière qui s'était notamment illustrée durant les deux sièges comme un organe de mobilisation populaire, la gazette avait un déficit flagrant de patriotisme. Son étiquette de gazette de propagande impliquait donc pour ses rédacteurs afrancesados un combat quotidien pour montrer à la fois leur hispanité intellectuelle et culturelle et, parallèlement, leur supposée indépendance rédactionnelle face aux gouvernants locaux. Contrairement à son adversaire de la résistance, la presse afrancesada, autant par la faute de ses auteurs que par la teneur de son discours, ne sut ou ne put jamais toucher véritablement son public. Faute de vulgarisateurs, de médiateurs ou plus simplement de transmetteurs du discours vers ses destinataires, comme le prêche des curés et les appels populaires pouvaient l'être, le discours afrancesado devait rester confiné aux débats des élites locales comme son prédécesseur ilustrado.

Enfin, n'oublions pas que la gazette se construisait dans les premières années de sa parution pro-napoléonienne autant comme un système planifié d'endoctrinement qu'en réaction aux événements, qu'ils soient internes au régime (place du conflit entre Joseph et Napoléon sur la propriété des régions du nord de l'Ebre à partir de 1810-1811) ou externes à ce dernier (résistance espagnole, alternances de défaites ou victoires militaires européennes).

Toutefois, bien que la dominante propagandiste fut tout au long des années d'occupation la raison même d'exister de cette presse, celle-ci diminua avec la pacification de la zone nord de l'Ebre, suite à la campagne obsidionale, transformant la gazette afrancesada bien plus qu'en un simple instrument du système napoléonien, en digne héritière de la presse de la «Ilustracion ».

La gazette nationale de Saragosse ou le retour de l'Illustracion. D'abord un journal de proximité.

31 Plus qu'une gazette propagandiste, la gazette nationale fut pour son rédacteur une gazette afrancesada, juste continuité ou résurgence d'un passé pas si lointain. C'est ainsi que Miguel Ased proposait à ses lecteurs non seulement un journal de proximité, mais aussi un journal éclairé se plaçant en digne héritier de Nipho et de Escrich. Comme ses descendantes du XVIIIe siècle, la gazette nationale de Saragosse répondait avant tout aux exigences de la pluridisciplinarité. Passant allègrement des affaires internationales aux variétés culturelles, englobant aussi bien des discours scientifiques que des représentations théâtrales, elle concluait plus modestement en se muant en journal de proximité par ses petites annonces locales, à la fois attrait du public et source 
complémentaire de rentrées d'argent. Comme journal dit de proximité, la gazette servait de diffuseur d'informations locales à destination principalement d'un public de notables ou de commerçants, bref à l'intention de ceux qui avaient non seulement les moyens de l'acheter, mais aussi les capacités de la lire.

Parlant du Diario de Zaragoza des dernières années du XVIIIe siècle, Blanco Murillo dans l'introduction de son ouvrage définissait son existence comme suit : "Le journal de Saragosse va être tout au long de son histoire un reflet du comportement et des attitudes d'une élite politique locale, d'une oligarchie d'une ville qui possédait une importante fonction de marché, une zone où le poids de la bourgeoisie commerciale, industrielle et agraire était conséquent $»$.

Sitôt le Diario disparu, la gazette prit donc le relais, combattant par son existence même l'image d'une Espagne d'Ancien Régime figée dans son archaïsme économique et sa foi dévote. Dans sa volonté première du ser util (être utile), la gazette offrait à ses lecteurs trois types d'encarts que nous pourrions qualifier de publicitaires. Les avis ou "avisos » et les « anuncios", les ventes et les livres.

Les avis et autres annonces tout d'abord, puisque les plus nombreux, se situaient comme le reste des encarts en dernière page de presque chaque gazette. Dans les annonces commerciales, l'on passait aisément de la location d'un appartement à des offres de service de porteurs ou de femmes se proposant comme nourrices. À côté de celles-ci se trouvaient les annonces dites légales fournissant tout aussi bien de nombreux postes publics vacants que des avis concernant les dernières réglementations. La fonction de journal de proximité se doublait pour l'occasion avec celle d'annonceur légal du gouvernement ${ }^{13}$.

Passons aux ventes. Elles aussi étaient fréquentes dans la gazette, leur nombre augmentant sensiblement avec la pacification de la région. À l'égal des annonces et autres avis, les ventes d'appartements ou de voitures à quatre roues proliférèrent dans une gazette qui se voulait au-delà du journal propagandiste, un outil de tous les jours à l'intention de ses lecteurs. Enfin, attardons-nous quelques instants sur la rubrique consacrée aux livres afin d'en déceler les particularités. Occupation oblige, la première allusion faite à la vente de livres concernait la parution d'un nouveau dictionnaire français-espagnol. Suivait la liste de toutes les bonnes librairies où l'on pourrait le trouver. Puis, chose rare, le même article, mais cette fois dans la langue de Molière. De la même manière, notons la présence d'un ouvrage traitant du change entre les monnaies françaises et les reales vallones espagnoles ou encore d'un prospectus offrant " des cours de langue française avec autorisation par Monsieur Thomas, ex consul de France aux États-Unis ». S'agissant de l'unique cas de bilinguisme présent dans notre gazette, nous ne pouvons une nouvelle fois que constater les différences de rédaction entre les gazettes afrancesadas, celle de Saragosse marquant, ici, sa différence avec la gazeta oficial de la Navarra où le bilinguisme était à l'origine même du journal ${ }^{14}$.

Ainsi, la guerre, loin d'être un frein au développement commercial, créait ses propres règles, choisissait ses partenaires et engendrait par-là même sa propre économie. Comme reflet des attentes d'une partie de la société aragonaise, la gazette de proximité nous offrait l'image, certes tronquée, mais pour partie existante, d'une Saragosse relativement active eu égard aux conséquences des deux sièges de 1808-1809. C'est ainsi que tout naturellement, le contexte politico-économique aragonais aidant, va apparaître lors des années 1811-1812 ce que nous pourrions appeler la résurgence d'un 
véritable journalisme illustré aragonais à travers la place grandissante réservée aux variedades.

Une gazette « ilustrada ».

37 Symboliques à la fois d'un certain retour au calme, mais aussi d'une volonté pour quelques-unes de dépasser les contraintes du quotidien, ces variedades et la place qu'elles occupaient, furent à l'image des espoirs de leurs auteurs. Ceux-ci, croyant dans une possible stabilisation politique à moyen terme, se ré-accaparaient tout simplement les fondements culturels de leur classe sociale «ilustrada » que les premières années de guerre avaient mis en suspens. Ainsi, au même titre que l'afrancesamiento est une résurgence envisageable de l'Ilustracion, nous allons à présent voir que la gazette nationale de Saragosse ne fut pas seulement un organe de propagande, mais avant tout une gazette illustrée ayant su s'approprier les talents de la gazeta et du diario du XVIIIe siècle.

Pour ce faire, le rédacteur va bénéficier d'une plus grande place laissée à la culture et aux sciences par la censure napoléonienne. Une étude comparative des gazettes nationales de Saragosse des années 1810 à 1812 nous permet en effet de constater une évolution sensible de la ligne rédactionnelle de la gazette au cours de ces trois années et ce en trois phases distinctes. Lors de la première, du début de l'année 1810 au milieu de la même année, la gazette ne laissait qu'une moitié de page de ses quatre pages totales à l'intention des annonces ou des variedades. Puis la seconde phase voit une légère augmentation de ces dernières avec les victoires de Lérida, de Tortosa et de Tarragona. Enfin, après la seconde moitié de l'année 1811, la zone comprenant l'Aragon, la Catalogne et presque tout le Levantin une fois pacifiée, la gazette se muait en véritable journal illustré. Ce ne sont plus alors une, mais plusieurs pages d'Ilustracion qui formaient avec les affaires internationales la composante majeure de la gazette.

D'ailleurs, dans celle du dimanche 1er avril 1810, le rédacteur Miguel Ased anticipait déjà ce que serait la future vocation de sa gazette dans ce qui ne représentait alors qu'un encart en dernière page. Sous le titre «Literatura » et à destination de «los sabios aragoneses ", Ased expliquait à la fois sa croyance dans le nouveau gouvernement (propagande oblige), mais aussi dans le futur de l'Espagne ou plutôt dans le retour de son glorieux passé.

"Quelques légers éclairs du bon goût aragonais se manifestèrent dans les périodiques qui parurent durant les années 1798 et 1799 du siècle passé [...] le Diario et le Seminario de Saragosse purent sans honte, s'installer à côté des plus réputés d'Espagne. Aussi futiles dans leur finalité qu'intéressants dans leur origine, ils moururent d'une mort indigne, sans que personne ne s'attache à les ressusciter ».

S'installant comme la synthèse du Diario, journal de proximité à destination de la bourgeoisie commerçante saragossaine et du Seminario de Saragosse, périodique illustré et encyclopédiste par excellence, la gazette, selon les dires de son auteur, proposait alors un retour pur et simple de l'Ilustracion aragonaise.

«Les malheurs de la guerre frustrèrent les louables desseins de quelques lettrés qui travaillaient à l'élaboration d'un nouveau périodique. Le vacarme des canons fit se taire la douce voix des filles d'Apollon et de Minerve, des fils des fertiles bords de l'Ebre, à la vue du furibond Dieu des batailles ».

41 Puis revenant à de plus concrètes réflexions, il affirmait :

«Le calme rétabli en Aragon, sous les auspices d'un gouvernement éclairé, il semblerait que le temps soit venu de ressusciter dans cette province le goût des belles lettres et d'établir grâce à elles, les principes d'une solide érudition [...] La 
grande œuvre qui se préméditait depuis le commencement de la gazette nationale de Saragosse ».

Pour conclure, poussé par sa mission de régénération des esprits, Ased interpellait une nouvelle fois les savants et autres érudits aragonais :

«Savants aragonais ! Rejoignez-nous, et l'Aragon verra renaître avec plus d'avantages les temps heureux de son Ilustracion ».

En tant qu'héritière des gazettes du XVIIIe, la gazette nationale de Saragosse fit alors sienne des thèmes majeurs de l'Ilustracion aragonaise. Ces variétés, que nous pourrions qualifier d'utilité et d'éducation publique, ne se limitaient pas seulement à la généralisation du glucose ou aux avantages nutritifs de la patate, mais pouvaient toucher de multiples domaines tant le rédacteur Miguel Ased montrait dans ses articles un éclectisme digne d'un encyclopédiste du siècle passé. S'insurgeant une semaine contre les peurs de ses concitoyens face aux pluies extraordinaires, il n'hésitait pas la semaine suivante à parler des méfaits de l'Inquisition, ou bien encore de vaccinations ou de cautérisations des morsures animales. Cependant, bien que l'on puisse louer de tels efforts, il est peu probable que l'impact suscité par de tels articles outrepassa le cadre d'une minorité afrancesada.

Conscient, comme les ministres afrancesados, que l'adhésion à son discours passait par un changement des mœurs espagnoles, $M$. Ased, afin de soutenir le nouveau système éducatif, articula pendant quelques temps ses variedades autour de ce thème. Regroupant ses articles sous le titre de "discurso sobre la educacion", notre rédacteur, accompagné d'autres auteurs occasionnels, reprenait les plus beaux arguments de Jovellanos en y apportant une pointe d'ironie anti-cléricale et de libéralisme économique ${ }^{15}$.

Enfin, la fusion entre l'afrancesamiento et l'Ilustracion aragonaise pouvait prendre une tournure encore plus concrète. Sous le titre « Première Junte du Canal Impérial d'Aragon et Royal de Tauste » était repris le discours inaugural fait par d'Hautefort à destination des nouveaux membres de ladite junte. Afin de symboliser la parenté des afrancesados avec les hommes de l'Ilustracion aragonaise, ce dernier dressait dans son préambule l'éloge de R. Pignatelli, illustre fondateur du canal. Puis il dévoilait les grands axes de travail de la junte devant répondre aux exigences du développement agricole local. Or, là encore, il n'était point question de nouveautés mais uniquement d'une reprise de la politique de la Réal Sociédad de Los Amigos Del Pais, incarnation par excellence du règne de Charles III ${ }^{16}$.

Pour résumer, nous pourrions dire que de simple relais d'une propagande étrangère jusque dans les années 1809-1810, la gazette sut, à partir de 1811, se convertir en véritable journal illustré capable de répondre aux exigences de proximité d'un journal local en même temps qu'aux aspirations d'une minorité érudite. Pour ce faire, en équilibristes du discours qu'ils étaient, nos rédacteurs se devaient de réduire, chaque fois qu'ils le pouvaient, les oscillations perceptibles pour leurs lecteurs entre deux mouvements contradictoires. À la fois changer les mentalités de leurs compatriotes pour les faire adhérer au nouveau régime, tout en montrant leur filiation avec une Ilustracion typiquement espagnole bien plus importante à leurs yeux que leur afrancesamiento au sens premier du terme. En tant que reflet de l'idéal ilustrado d'une partie des élites locales, la gazette ne pouvait, par conséquent, que nous interroger sur l'afrancesamiento en général et sur son contenu provincial en particulier ${ }^{17}$. 
Étude des gazettes afrancesadas et nouvelle perspective de l'afrancesamientoQuestion de définition

Tout d'abord, revenons sur le sens et l'origine même du mot afrancesado. Embrouillé jusque dans sa définition, ce terme peut se traduire de deux manières d'apparences complémentaires et pourtant bien différentes. La première caractérise l'afrancesamiento comme un courant intellectuel exagérément français. Employé à l'origine pour évoquer une gallomanie bouffonne et légère, l'afrancesamiento s'est ensuite attaché aux Espagnols du XVIIIe ayant pioché des doctrines politiques en France par pur mimétisme. La seconde, quant à elle, consiste à définir l'afrancesado comme "Le" collaborateur espagnol du régime napoléonien. On parle alors d'afrancesamiento politique pour le différencier du culturel. M. Artola ${ }^{18}$ va même plus loin en affirmant que son aspect culturel appartenait plus aux libéraux en opposition au domaine politique des dénommés afrancesados collaborateurs. Cependant, au même titre qu'une autre, cette définition est contestable. Pourquoi en effet, comme le dit M. Artola, les libéraux seraient-ils des afrancesados culturels plus que les afrancesados eux mêmes ? N'y a-t-il pas là un contresens évident? Au surplus, sous prétexte que les libéraux puisèrent leur inspiration dans la philosophie des Lumières, doit-on leur laisser l'apanage de l'hérédité culturelle ? Et si oui, de quelle hérédité culturelle parle-t-on?

Car l'afrancesamiento n'est pas une idéologie bien définie, toutes les idées venues de France ne constituant pas un bloc homogène. Le caractère multiforme de l'influence française (idéal révolutionnaire, des Lumières ou encore impérial) fait donc de l'afrancesamiento une parenté aléatoire plus qu'un contenu tangible. En suivant toujours la définition en vigueur, qu'il soit simple collaborateur ou ministre, l'afrancesado pouvait balancer du jacobinisme le plus excessif au despotisme éclairé ${ }^{19}$. Mais alors, que veut dire afrancesado? Peut-on raisonnablement parler d'hétérogénéité au sein des afrancesados sous prétexte d'englober sous ce terme, jacobins, néo- ilustrados, absolutistes et libéraux, du simple fait de leur adhésion au nouveau régime ? Le terme d'afrancesado suscitant toujours le débat, évitons donc d'y recourir systématiquement et optons plutôt, pour l'époque qui nous concerne, pour celui de collaborateurs comme dans tous les autres cas d'occupation.

Reste cependant l'épineuse question du double afrancesamiento culturel et politique prôné par $\mathrm{M}$. Artola comme alternative à cette hétérogénéité. Pour y répondre nous nous appuierons sur les variedades de la gazette qui, à l'instar des pages culturelles de ses consœurs françaises, dépassaient de loin le simple cadre culturel pour aboutir à la création d'un véritable discours politique.

Les « variedades » en question

50 Selon A. Cabanis, "c'est sous cette rubrique que les gazettes font preuve de personnalité. Dans les comptes rendus de livres, dans les jugements portés sur les pièces de théâtre, elles parviennent à glisser quelques traits, quelques allusions qui permettent de définir sinon leur couleur politique du moins leur nuance idéologique $»^{20}$.

51 Suivant ce principe et sous le titre " Variétés : sur la décadence littéraire espagnole », le rédacteur Miguel Ased nous proposait ainsi sa propre définition de l'afrancesamiento culturel :

«Quiconque comparera l'état des Lettres en Espagne au XVIe et XVIIe avec l'état actuel, remarquera une différence digne de se lamenter et de contempler les rapides progrès qu'elles ont faites dans les autres nations d'Europe [...] Ont disparus 
les Quijotes, le théâtre critique, et tous les livres méritant d'être traduits dans toutes les langues cultes [...] Entre les différentes choses qui ont concouru à cette dépravation du mauvais goût, origine de la décadence de notre littérature, ont peu compter avec la coupable adhésion des Espagnols pour tout ce qui n'est pas espagnol et la faute de choix quant il s'agit de s'enrichir d'œuvres étrangères [...] Il y eut l'époque du latin, et maintenant il n'y a pas autre chose que des livres remplis de gallicisme, parce que leurs auteurs, tous plongés dans la langue française, ne daigneront prendre dans leurs mains, un dictionnaire de l'académie, ni lire deux pages de Granada ou d'Argensola. »

52 Puis, après cet hymne à la gloire de l'Espagne que l'on aurait pu aisément prêter à un conservateur du camp d'en face, le rédacteur revenait à des réflexions moins passionnées : «Loin de nous de vouloir réprouver l'étude des langues et la lecture d'écrivains étrangers. Ce ne fut jamais autant nécessaire qu'en ce moment. Mais n'oublions pas nos classiques, notre riche langue et surtout espérons qu'il y ait plus de discernement dans le choix des traductions $»$.

Plaidoyer pour la redécouverte d'une véritable culture espagnole, ce texte représentait un contre-pied magnifique à la vision restrictive de l'afrancesamiento. Les afrancesados comme les ilustrados leurs prédécesseurs étaient donc bien plus que de vulgaires moines copistes de l'Esprit des lois ou de l'Encyclopédie. Si la notion d'une Espagne décadente revenait comme une récurrence du discours afrancesado, elle se voyait compenser par une hispanité marquée et très éloignée de la francisation excessive que l'on prête à tort à ses concepteurs. Empreint de civilisation gréco-latine, Ased rêvait d'un nouvel âge d'or de l'Espagne allant même jusqu'à critiquer le gallicisme et les importations culturelles dont les Espagnols étaient coupables. Pour Ased, l'Espagne déclinait, car ses enfants cherchaient ailleurs ce qu'elle avait en elle. Et notre rédacteur, loin d'une adhésion aveugle aux idées venant de l'étranger, espérait simplement que les Espagnols accompliraient à leur tour le lent chemin menant leur pays dans le rang des nations « cultes ».

D'un point de vue plus général, cette définition de l'afrancesamiento culturel de Ased n'est pas sans poser le problème de la notion de modèle. En effet, loin d'être un ensemble compact de doctrines, l'Ilustracion, comme l'afrancesamiento plus tard, ressemblait plus à un mouvement global d'aspiration au progrès mélangeant conservatisme politique et libéralisme économique et social. La France qu'ils admiraient n'était ni un territoire ni une librairie et encore moins un empereur, mais plutôt ce pays «culte» qui leur offrait les voies d'une renaissance culturelle et politique. La France, comme l'Angleterre d'ailleurs, n'était pas un pays à copier, mais bel et bien un modèle à suivre ${ }^{21}$.

Nous opterons, par conséquent, pour la définition d'un afrancesamiento correspondant à un lent syncrétisme culturel et politique plutôt qu'à celle plus traditionnelle d'un courant idéologique adepte du plagiat culturel pur et simple ou de la collaboration politique aveugle. C'est d'ailleurs le paradoxe que relève L. Dupuis ${ }^{22}$ d'une définition d'un afrancesamiento importateur de doctrines étrangères par simple mimétisme, alors même que la ou les doctrines importées (les Lumières, si l'on peut résumer les Lumières à un seul courant de pensée) font la part belle à l'esprit critique et au raisonnement. Au surplus, limiter l'afrancesamiento à un conglomérat d'importations françaises sur le territoire espagnol revient à extérioriser le mouvement dans son ensemble, à nier le syncrétisme dont il est le fruit et par ricochet à l'écarter de la riche histoire des mentalités espagnoles ${ }^{23}$. Comme si le particularisme espagnol resurgissait dans une 
lecture téléologique de l'histoire de l'Espagne du XIXe au regard de ses voisines européennes en voie de démocratisation.

Toutefois, bien que nuançant la vision de M. Artola, L. Dupuis n'en distingue pas moins le fait culturel $\mathrm{du}$ fait politique, à trop vouloir présenter le politique comme une conséquence naturelle et inévitable du premier nommé. Ce serait, selon lui, la porte ouverte au discrédit des Lumières en Espagne en les entachant de trahison politique, comme le fit en son temps Melendez Pelayo. Mais, à l'inverse, il faut se garder de nier un rapport évident entre les deux afrancesamiento, les variedades, par leur existence même, constituant un exemple clair de cette collision entre le politique et le culturel ${ }^{24}$. De même, pendant longtemps l'afrancesamiento culturel provincial ne fut envisagé que sous l'angle du gallicisme vestimentaire ou architectural, comme si la transition vers l'adhésion idéologique des Espagnols n'avait pas touché la province, mais exclusivement les élites madrilènes. Or, à vouloir opposer trop systématiquement l'afrancesamiento culturel des élites provinciales à celui plus idéologique des Madrilènes, ne dédouanons nous pas de facto les provinces espagnoles de l'acte de collaboration tout en le réservant à une minorité castillane, ce que la tendance régionaliste de la recherche historique actuelle ne peut contredire?

$\mathrm{Au}$ demeurant, le risque, en morcelant ainsi ces deux afrancesamiento, serait d'extérioriser le politique et de ne pas le percevoir comme la continuité d'une idéologie ayant, aussi, existé sous Charles III. Enfin, assimiler l'afrancesamiento politique à une adhésion au régime napoléonien est une erreur. Cela revient à ignorer les divergences quant au devenir politique de l'Espagne entre Napoléon et Joseph et à rejeter la volonté première des afrancesados de maintenir, en l'état, l'intégralité de leur territoire et de leur identité nationale ${ }^{25}$.

59 Accepter la paternité d'un mouvement afrancesado de collaboration ne dissociant pas forcément le culturel du politique comme une résurgence d'une idéologie nommée Ilustracion n'implique nullement de contester les visions traditionnelles de l'historiographie espagnole, mais d'apporter un questionnement nouveau. Le cœur de nos travaux de recherche se trouvera donc en province pour savoir s'il existait, par delà la propagande napoléonienne et les écrits de la presse madrilène, un véritable afrancesamiento culturel et politique dans la presse propagandiste provinciale.

De l'intérêt d'une étude comparative

60 S'il nous fallait résumer en quelques lignes l'intérêt d'une telle étude globale, nous dirions que c'est par sa situation même de carrefour que cette étude vaut d'être menée. Pour simplifier, l'analyse de la presse afrancesada veut s'installer à la confluence de trois orientations historiographiques majeures : celle du renouveau de l'histoire de la presse espagnole, d'une histoire sociale du politique de l'Espagne et d'une histoire napoléonienne moins ethnocentrique car située hors de France.

61 Examinons donc quelques-unes des pistes que notre étude devra éclaircir pour parfaire notre connaissance de l'afrancesamiento.

Tout d'abord essayer dans le cadre d'une analyse globale de percevoir une cohérence d'ensemble entre ces gazettes afrancesadas. Pour ce faire, prenons garde en premier lieu à la construction théorique de la propagande face à la réalité quotidienne de l'occupation. Là encore, il s'agit plus d'un assemblage fluctuant en fonction des événements qu'autre chose. Mais si adaptabilité aux circonstances il y a, quelles sont alors les lignes propagandistes communes à toutes les gazettes ? Les thèmes sont-ils 
différents, omis, voire accentués, en fonction du lieu ou des personnes ? Qui rédige et surtout pour qui et pourquoi ? De même, les publics touchés sont-ils identiques que l'on soit en Catalogne ou en Aragon? Autant de questions qui, à n'en pas douter, mériteront réflexions.

À travers les permanences, mais aussi les différences, entre les gazettes afrancesadas, ce sont ensuite les tensions internes propres à chaque région occupée qui se feront jour à travers des rapports de forces fluctuant en fonction des hommes comme des circonstances ${ }^{26}$.

Évitant une conception trop téléologique de l'histoire politique produite par la Guerre d'Indépendance, nous chercherons plutôt à affiner notre vision par une grille de lecture des tendances en rapport avec les choix qu'ont dû faire les acteurs dans le feu de l'action. Traditionnellement, la scission parmi les ilustrados entre libéraux et afrancesados n'est pas perçue comme le fruit du hasard, mais d'un choix entre l'orientation révolutionnaire et le réformisme politique. Là encore l'analyse est pertinente, mais incomplète. Quid des rivalités entre familles ? Quid des différences générationnelles entre afrancesados collaborateurs et libéraux résistants pour expliquer leur choix ? Quid, enfin, des lignages, des réseaux et du clientélisme si utiles pour comprendre la genèse de l'État espagnol des XVIe et XVIIe siècles et pourtant si peu présents lors de l'analyse des clivages politiques de la Guerre d'Indépendance? C'est d'une étude sociale dont il sera ici question que cela soit en amont de la Guerre d'Indépendance comme en aval par les connexions de l'afrancesamiento journalistique et de ses acteurs avec le «Trieno Liberal».

65 À l'échelle de la presse, il conviendra de redéfinir la place du journalisme afrancesado dans le cadre naissant d'une première presse d'opinion en Espagne. Si la presse napoléonienne fut docile et souvent sans relief, celle de ses voisins européens était d'un tout autre acabit. Dès lors, où positionner un journalisme afrancesado tiraillé entre les obligations de l'occupation, les désirs de ses auteurs et la pression médiatique de ses adversaires. C'est à l'analyse d'une véritable révolution journalistique qu'il faudra s'attacher avec son corollaire d'interrogations. Ne retenons à titre d'exemple que l'épineux problème de l'anonymat des rédacteurs. Correspond-t-il, comme nous pourrions le penser, à une crainte ou à une honte de leur part ? Pas forcément ! Car n'oublions pas que les noms de rédacteurs se retrouvaient quasi systématiquement dans les variétés culturelles dont la consonance politique a été démontrée, faisant de la signature de leur auteur un engagement bien plus important qu'il n'y paraît.

Dernière orientation, sortir l'étude de la presse napoléonienne hors des frontières nationales. «Au fur et à mesure que l'on s'éloigne de Paris, l'importance des nouvelles politiques s'accroît » selon Cabanis ${ }^{27}$. La question sera donc de savoir si cette presse afrancesada, spécifique car distante du pouvoir central, fut à l'identique de sa consœur française ou non. Comparer avec la presse française nous permettra, à n'en point douter, de voir où revoir les notions de centre-périphérie ou de propagande dans un cadre plus large et donc forcement plus complexe. À plus grande échelle, la propagande dans ses incohérences comme dans son unité offrira alors de multiples questionnements notamment dans le rapport changeant, via la presse d'occupation, des élites européennes à Napoléon, d'une part, et à l'idéal des Lumières et de la Révolution, d'autre part.

67 On le voit, les champs de recherche sont nombreux, allant de la linguistique (utile par exemple pour voir le degré d'acculturation des afrancesados ou même la présence des 
rédacteurs français dans certaines gazettes), à l'étude des concepts politiques naissants en passant par l'analyse du rôle de la presse dans l'élaboration d'un nouveau langage politique et plus généralement dans la politisation des masses. Car comme le disait Napoléon : «Quatre périodiques feront toujours mieux que 100000 soldats ». Or, si son analyse était des plus juste, la mise en œuvre ibérique fut loin du résultat escompté, les Espagnols étant bien plus qu'un corps d'armée à flatter, une nation naissante qu'il fallait convaincre.

\section{NOTES}

1.A. Cabanis, La presse sous le Consulat et l'Empire, Paris, Société des études robespierristes, 1975, p. 67.

2.Dans J. Tabar López, Los famosos traidores. Los afrancesados durante la crisis del Antiguo Regimen (1808-1833), Madrid, Ed. Biblioteca Nueva, 2001. Régulièrement, de nouvelles gazettes sont ajoutées à l'inventaire des gazettes afrancesadas. Le travail d'inventaire (en cours) est donc primordial pour connaître l'intégralité de notre thème d'étude. 3.Parmi celles-ci, notons : L. Puig i Oliver, Notes sobre la premsa napoleónica a Girona, Anales del Instituto de Estudios Gerundenses, 22, 1974-1975. Ramos Demetrio, La técnica francesa de formación de opinión desplegada en Barcelona (1808-1809), Estudios sobre la guerra de la Independencia, Zaragoza, Institución Fernando el Católico, 1965. J. Tabar López, «Los métodos de captación del régimen josefino: la propaganda afrancesada », dans Gil Novales, A (coord.), La revolución liberal española en su diversidad peninsular (e insular) y americana.

4.J. Blazco Ijazo, Historia de la prensa zaragozana (1683-1947), Zaragoza, Ed. El Noticiero, 1947, et Duenas Labarias, Historia del periodismo en Aragon, Zaragoza, Ed. Diputacion de Zaragoza, 1990.

5.Sa devancière résistante « la gazette de Saragosse » sous direction espagnole était vendue aux bénéfices de Nuestra Senora de Gracia et conçue dans l'imprimerie Moreno. Notons simplement comme symbole de la permanence du journalisme aragonais que l'imprimeur (Moreno) et certaines librairies (Ruiz) seront toujours dépositaires du journalisme aragonais que ce soit avant et pendant les sièges de la ville, durant l'occupation et après le départ des Français d'Espagne.

6.J. J. Sánchez Aranda, « Napoleón y la prensa afrancesada en España », dans Les Espagnols et Napoléon, op. cit.

7.D'après les comptes d'A. Sevil, les coûts d'impression, de rédaction et de papiers du 14 août 1809 au 31 décembre 1809 s'élèvent à 9609 rv. Puis à $15489 \mathrm{rv}$ pour les 6 premiers mois de 1810 et à $11400 \mathrm{rv}$ pour les 6 derniers mois de 1810, soit un coût total de 36498 rv pour la période. Dans le même temps les recettes du 14 août au 31 décembre 1809 s'élèvent à $9585.24 \mathrm{rv}$, puis à $12205.18 \mathrm{rv}$ du $1 \mathrm{er}$ janvier 1810 au 30 juin 1810 et à 5 046.4 du 1er juillet au 31 décembre de la même année. Les recettes totales $(26837.12 \mathrm{rv})$ sont donc bien inférieures aux coûts sur cette période avec un écart de $9660.22 \mathrm{rv}$. Il s'agit donc d'un véritable maintien sous perfusion financière dont il est question, puisque seul le second semestre de 1809 est bénéficiaire. 
8.Le terme de résistance, conçue comme un bloc uni et opposé au système en place, existait néanmoins quand la gazette se référait à la Junte Suprême et à son allié britannique. Mais cette dernière devait apparaître suffisamment lointaine et distante pour ne pas susciter dans l'esprit des lecteurs une quelconque connexion avec les affaires d'Aragon.

9.La gazette nationale de Saragosse en diffusant ainsi les ordres du jour et autres félicitations, outre le fait de saboter le moral des adversaires, facilitait le transfert d'information vers la France, donnant à l'opinion publique française l'image que Napoléon voulait de sa Grande Armée.

10.Il faut savoir que de nombreuses lettres de soldats espagnols déportés en France permettaient à ces derniers d'obtenir, contre quelques lignes de trahison, de conséquentes améliorations de leurs conditions de détention souvent rudimentaires. 11.Seule une étude comparative de toutes les gazettes afrancesadas permettra de déceler ce qui relève de la propagande des Tuileries, de Madrid ou des acteurs locaux. 12. Contrairement à J. J. Aranda, je considère que Ferdinand fut relativement épargné par la presse afrancesada. Il n'est cité en trois années qu'à cette unique occasion. Pour ce qui concerne l'affaire de Valençay, j'ajouterais que les attaques ne sont pas directes et touchent plus à la perfidie anglaise qu'à la faiblesse (par sa soumission) du Prince. Ces critiques sont d'ailleurs très éloignées de celles plus virulentes qu'eurent à subir les autres grands acteurs de la résistance espagnole comme le marquis de la Romana ou Wellington.

13.Parmi les postes offerts, l'on pouvait trouver régulièrement des propositions de places au sein de l'Audiencia d'Aragon en remplacement d'un titulaire décédé. Bien que logique dans le cadre d'un simple changement de personnel ou d'une réorganisation administrative en temps de paix, la multiplicité de ces exemples de poste à pourvoir dans le domaine judiciaire symbolisait, encore une fois, l'incapacité de l'administration occupante à trouver des hommes de droit pour leurs postes vacants. En effet, dans l'un de ses avis publiés déjà à de nombreuses reprises et tiré de la gazette nationale de Saragosse $\mathrm{n}^{\circ} 22$ du jeudi 8 mars 1810 , était une nouvelle fois proposé un poste de rapporteur, libre depuis la mort de son propriétaire D. Mariano Garcia le 17 juillet 1809, soit quelque neuf mois en arrière.

14.De telles différences ne peuvent que nous interpeller sur les divergences rédactionnelles des gazettes, l'origine de leurs rédacteurs ou encore le public recherché. Le bilinguisme, par exemple, implique-t-il un rédacteur français ou espagnol et plus encore un public non exclusivement hispanique ? Est-il une constante des gazettes afrancesadas ou un cas isolé ? Tous les thèmes sont-ils dans les deux langues ou uniquement les annonces légales et autres variétés culturelles? Autant

d'interrogations nécessitant à leur tour une synthèse globale.

15.Avec l'émergence d'un discours illustré conséquent à partir de 1811, les variedades n'étaient plus, uniquement, le fruit du rédacteur du journal, mais de plus en plus une tribune collective de médecins ou d'avocats locaux. La gazette offrait à chacun un lieu de débat où l'esprit critique et les divergences d'opinion trouvaient, dans une certaine limite, leur place.

16.Celle-ci, créée en 1776 sur proposition de la municipalité aragonaise, regroupait en son sein toute l'élite aragonaise éclairée qu'elle soit bourgeoise ou aristocratique. Ses objectifs étaient centrés, en tant qu'émanation et relais provincial du gouvernement de Charles III, sur le développement de l'éducation, de l'agriculture et de l'industrie. 
17.Au passage, contrairement à ce qu'affirme $\mathrm{P}$. A. Blanco Murillo sur la gazette de Saragosse, l'autre journal de la ville, le Diario de Zaragoza, n'était pas le seul à répondre aux exigences des élites saragossaines en publiant annonces et autres avis. Selon cet historien, « les annonces locales étaient absentes durant toute la trajectoire de la gazette de Saragosse». Or, si la gazette de Saragosse puis la gazette nationale de Saragosse n'avait pas pour unique fonction de diffuser des annonces locales, il est faux d'affirmer qu'elles n'en proposèrent aucune. La volonté d'être utile n'était pas l'exclusivité du Diario de Zaragoza, loin de là. La gazette allait même plus loin dans cette logique en répondant avec d'autres arguments que ceux d'un journal de proximité uniquement. En outre cela distingue cette presse espagnole de la presse française qui subira concrètement les mises au pas des lois de 1810-1811.

18.M. Artola, Los afrancesados, Madrid, Ed. Alianza, 1989.

19.Voir à ce sujet l'analyse de l'hétérogénéité du mouvement afrancesado faite par Luis Barbastro Gil dans Los Afrancesados : Primera Emigracion Politica, Madrid, CSIC : Instituto de Cultura Juan Gil-Alberti, 1993.

20.A. Cabanis, op. cit., p. 105.

21.Notons encore une fois les différences avec la gazette officielle de Navarre où l'Angleterre était associée à l'Espagne dans le groupe des nations de second rang, alors que pour le rédacteur de la gazette nationale de Saragosse elle appartenait au clan des nations « cultes ». Qui plus est, ce discours afrancesado n'est pas sans aller, une nouvelle fois, en contradiction avec la propagande strictement napoléonienne dont l'anglophobie était une composante majeure.

22.L. Dupuis, «À propos d'afrancesamiento », Revue Caravelle, numéro 1, Toulouse, 1963. 23.Si l'on se limite exclusivement à l'afrancesamiento relevant d'une «néo-Ilustracion » tardive, c'est alors à la recomposition d'un mouvement politique long qu'il faudra s'atteler, en partant de l'époque de sa fondation, en passant par sa résurgence sous l'occupation napoléonienne pour finir par son influence notable dans l'époque du Trieno Liberal.

24.Distingue-t-on de la même manière les aspects culturels et politiques de l'américanisation des pays européens depuis 1945 ? Certes, Hollywood n'a que peu de liens avec le modèle politique ultra-libéral diffusé par les États-Unis. Toutefois, les connexions entre ces deux américanisations existent par l'intermédiaire d'un discours individualiste orienté sur le mythe du «self made man ». Bien évidemment, le degré d'absorption par chacun de ce discours est variable, mais il difficile d'envisager le cheminement de l'américanisation politique sans son pendant culturel. Au demeurant, la différence entre la perception qu'ont les historiens de l'américanisation et de l'afrancesamiento en tant que système d'acculturation démontre combien le second est encore entaché d'une trahison historique indélébile.

25.Là encore, seule l'analyse de toutes les gazettes afrancesadas nous permettra de savoir si, oui ou non, le discours afrancesado a subi des modifications avec les annexions de 1811. Celles-ci, dépeçant l'Espagne de ses régions les plus riches, ne furent acceptées ni par Joseph ni par son entourage. En conséquence, l'hypothèse d'un changement de perception de Napoléon par les rédacteurs afrancesados est envisageable même si celuici, une fois dans leurs articles, n'est pas explicite et relève plus des sous-entendus et du détail, propagande et contrôle militaire obligent.

26.À l'argument d'A. Cabanis stipulant que le rapport d'éloignement entre les organes de presse provinciaux et le pouvoir central favorisait une certaine marge de manœuvre et d'expression plus importante, je rajouterais une autre conclusion propre à l'Espagne 
occupée que notre étude aura pour fonction d'éclaircir. En effet, si la distance joue un rôle fondamental dans les libertés d'expression, il faut lui ajouter non seulement le poids du représentant de l'État central sur place (préfet en France et gouverneur en Espagne du Nord) mais aussi les rapports de force inhérents à toute situation politique instable. J'entends par là une triple dualité pour le cas qui nous concerne. Une dualité de direction politique à l'échelle du gouvernement central entre le gouvernement de Napoléon et celui de Joseph à Madrid. Une dualité nationale ensuite, mettant cette presse dans une situation de concurrence et de conflit avec la presse résistante. Une dualité, enfin, à l'échelle provinciale entre le pouvoir civil (majoritairement des Espagnols afrancesados) et le pouvoir militaire composé de généraux français.

27.A. Cabanis, op. cit., p. 67.

\section{RÉSUMÉS}

Février 1809, Saragosse "la résistante» tombait sous les coups de canons des armées napoléoniennes. L'occupation militaire était à peine commencée que la gazette nationale de Saragosse voyait le jour avec pour but évident de mettre à mal la résistance espagnole. C'est ainsi que pendant près de trois années, cette gazette fera, à l'instar de ses consoeurs afrancesadas, contrepoids aux gazettes « insurgées » dans une sorte de " guerre des presses ", modèle réduit de la Guerre d'Indépendance. Pour se faire, la gazette utilisera tous les moyens de la propagande napoléonienne au risque de produire un discours pluriel rempli d'incohérences et de contradictions. Toutefois, à partir de 1810, la gazette se muera progressivement de journal officiel en véritable gazette ilustrada. De cette résurgence du journalisme ilustrado aragonais et de son affiliation avec l'afrancesamiento devait découler un nouveau questionnement sur l'afrancesamiento provincial, dans sa définition comme dans ses enjeux.

\section{The National Gazette of Zaragoza : between collaboration and 'Afrancesamiento'}

In February 1809, Zaragoza the "endurant" fell to the canonfire of the Napoleonic armies. The military occupation had barely begun when the National Gazette of Zaragoza saw the light of day, overtly to counter Spanish resistance. Thus, for nearly three years, this gazette, like its afrancesadas siblings, acted as a counterweight to the "insurgent" gazettes in a sort of "press war" similar on a smaller scale to the war of independence. To that end, the gazette resorted to all the Napoleonic propaganda tactics at the risk of churning out inconsistences and contradictions. As from 1810, however, the gazette shed its role of official news-sheet and became a true gazetta illustrada. This resurgence of illustrated Aragon journalism, affiliated to afrancesamiento, led anew to a calling into question of provincial afrancesamiento, both in its definition and the issues at stake

\section{INDEX}

Mots-clés : Espagne, gazette nationale de Saragosse, propagande napoléonienne, Ilustracion, afrancesamiento provincial, presse espagnole, Aragon 
AUTEUR

FRÉDÉRIC DAUPHIN

Université Blaise Pascal - Clermont-Ferrand 